\title{
Integration of medical therapy and mechanical circulatory support in the management of acute heart failure
}

Patrick Horn, Malte Kelm, Ralf Westenfeld

Division of Cardiology, Pulmonology, and Vascular Medicine, Medical Faculty, University of Düsseldorf, Germany

Submitted: 10 January 2015

Accepted: 1 February 2015

Arch Med Sci 2016; 12, 6: 1317-1323

DOI: 10.5114 /aoms.2015.51700

Copyright $\odot 2015$ Termedia \& Banach

\section{Abstract}

Acute heart failure is still characterized by poor prognosis with high mortality. Diagnosis is based on clinical symptoms and hemodynamic measurements. Early coronary revascularization in cardiogenic shock complicating myocardial infarction improves outcome. The further contemporary therapeutic options in the management of acute heart failure are limited to a merely symptomatic effect with relief of dyspnea, reduction of volume overload and improvement of hemodynamic parameters by vasodilators (in hypertension) or inotropic and vasopressor agents (in hypotension). However, so far no medical therapy has been shown to positively affect clinical outcomes of patients with acute heart failure. Early identification of impending circulatory collapse coupled with rapid implementation of mechanical circulatory support may contribute to mortality reduction as a combined concept of the management of acute heart failure.

Key words: acute heart failure, cardiogenic shock, coronary revascularization, medical therapy, mechanical circulatory support.

\section{Introduction}

Acute heart failure (AHF) can arise as a complication in the acute setting of myocardial infarction and acute coronary syndrome (ACS), but today most often (> 75\%) AHF arises due to decompensation of preexisting chronic heart failure (CHF) [1]. Chronic heart failure is a growing burden on health care with a prevalence of $1-2 \%$ in western countries and a rising trend due to aging of the population and contemporary therapy leading to improved survival in patients following a myocardial infarction [2]. The prognosis of systolic CHF has constantly improved over the last 20 years with the advent of renin-angiotensin-aldosterone system (RAAS) inhibition, the $\beta$-adrenergic blockade and device therapy in combination reducing the actual 1-year mortality of $\mathrm{CHF}$ to $5-10 \%$. In contrast to $\mathrm{CHF}$, 1 -year mortality of patients admitted for AHF still remains devastating at $30 \%$, even exceeding $50 \%$ in cardiogenic shock $[3,4]$. The 10 -year old SHOCK-trial was the last randomized controlled trial demonstrating the prognostic impact of the innovative early reperfusion strategy of the infarct-related coronary artery in therapy of cardiogenic shock $[5,6]$. But besides that, the contemporary therapeutic options in the management of AHF are limited to merely symptomatic improvement of hemodynamic parameters, reduction of volume overload and relief of dyspnea without

\author{
Corresponding author \\ PD Dr. Ralf Westenfeld \\ Division of Cardiology, \\ Pulmonology, \\ and Vascular Medicine \\ University Duesseldorf \\ Medical Faculty \\ Moorenstr. 5 \\ D-40225 Düsseldorf, \\ Germany \\ Phone: +49 21118800 \\ Fax: +49 2118118812 \\ E-mail: ralf.westenfeld@med. \\ uni-duesseldorf.de
}


prognostic benefit. In fact, no medical therapy has been shown to positively affect clinical outcomes of patients with AHF. As dyspnea is the leading burden for most patients with AHF, relieving dyspnea remains an important therapeutic goal. In addition to that, further goals in the management of AHF should be to prevent readmission and to improve mortality. Recently, new approaches in the management of acute heart failure have arisen, and involvement of these in the management might lead to achieving these goals.

First, every episode of AHF induces further damage not only to the heart, but also to other organic systems (kidneys, liver, brain), and accelerates the progression of the chronic disease leading to an increase in mortality of further acute cardiac decompensation.

Second, AHF that is hemodynamically non-stable (cardiogenic shock) induces microcirculatory disorder and systemic inflammatory response syndrome (SIRS), leading to multiple organ dysfunction syndrome (MODS). The progression to MODS seems to be one major determinant of the prognosis of cardiogenic shock. Therefore, prevention of MODS presents a new goal in the management of cardiogenic shock which might improve the prognosis of AHF.

Table I. Etiologies and precipitants of acute heart failure

- Tachycardia (atrial fibrillation/flutter, ventricular tachycardia)

- Bradycardia (higher degree AV block)

- Hypertensive episode

- Volume overload/abrupt stopping or reduction of the preexisting diuretics

- Deterioration of renal function

- Acute myocardial infarction

- Acute myocarditis

- Progression of valvular heart disease

- Acute dysfunction of prosthetic valve

Table II. Symptoms and signs of acute heart failure

\begin{tabular}{|c|}
\hline Symptoms \\
\hline $\begin{array}{l}\text { - Dyspnea } \\
\text { - Tachypnea } \\
\text { - Orthopnea } \\
\text { - Cough } \\
\text { - Fatigue } \\
\text { - Distress, anxiety }\end{array}$ \\
\hline Clinical signs \\
\hline - Third heart sound (S3) \\
\hline $\begin{array}{l}\text { Predictive for wet state vs. dry state: } \\
\text { - Peripheral edema } \\
\text { - Weight gain } \\
\text { - Rales on auscultation (possibly with wheezing) } \\
\text { - Jugular venous distension }\end{array}$ \\
\hline $\begin{array}{l}\text { Predictive for cold state vs. warm state: } \\
\text { - Signs of inadequate systemic perfusion (pale color, } \\
\text { peripheral cyanosis, altered mental status, oliguria) }\end{array}$ \\
\hline
\end{tabular}

Early identification of impending circulatory collapse coupled with rapid implementation of mechanic circulatory support (MCS) may normalize cardiac output, prevent the progress to MODS and may contribute to mortality reduction $[7,8]$.

The purpose of this present paper is to review the current state of knowledge about AHF. The review focuses on the contemporary therapeutic options integrated in a concept including medical therapy and mechanical circulatory support which might improve the prognosis of patients with AHF in the future when tailored to the appropriate patient at the appropriate time.

\section{Symptoms, signs and diagnosis}

The heterogeneous etiologies and triggers of AHF are summarized in Table I. Acute heart failure with its life-threatening symptoms requires immediate therapy; thus diagnosis and therapy need to be performed simultaneously. Acute heart failure is diagnosed based on a focused patient history and clinical examination. Regardless of precipitant or underlying etiology, pulmonary congestion due to elevated ventricular filling pressure and left-sided heart failure is a classical finding in AHF. This congestion is manifested in symptoms such as dyspnea and rales and, in the case of right heart involvement, in edema and weight gain. Fatigue is a less specific symptom of critically reduced cardiac output. Characteristic symptoms and signs of AHF are summarized in Table II, and an overview about the initial tests is presented in Table III. In this setting an audible $\mathrm{S} 3$ or orthopnea has a positive predictive value of $61 \%$ or $66 \%$ respectively for indicating an elevated pulmonary capillary wedge pressure (PCWP > $22 \mathrm{~mm} \mathrm{Hg}$ ) [9]. Serum concentrations of B-natriuretic peptide (BNP) and its inactive precursor N-terminal pro-BNP (NT-pro BNP) can be employed as an additional approach for ruling out AHF when clinical uncertainty exists. For patients presenting with acute onset or wors-

Table III. Tests for diagnosis and assessment of acute heart failure

- Pulse oximetry, arterial blood gas analysis

- Blood pressure

- Electrocardiogram

- Chest X-ray

- Echocardiography (global and regional systolic ventricular function, diastolic ventricular function, valvular diseases, pericardial diseases)

- Laboratory (troponin if ongoing ischemia is suggested, BNP, serum electrolytes, creatinine, blood urea nitrogen, hepatic enzymes, international normalized ratio)

- Invasive hemodynamic monitoring in patients with persistent symptoms/uncertain hemodynamics (Swan-Ganz catheter)

- Coronary angiography 
ening of dyspnea, the exclusion cut-off point is $100 \mathrm{pg} / \mathrm{ml}$ BNP or $300 \mathrm{pg} / \mathrm{ml}$ NT-pro BNP, respectively $[10,11]$.

Acute coronary syndrome precipitating AHF should be identified by medical history, electrocardiogram and cardiac troponin testing. Noteworthy, elevated troponin levels are common in AHF in the absence of primary ACS and related to endocardial malperfusion (supply-demand mismatch) and cytosolic troponin release. Thus, identifying ACS may be difficult but remains important since treatment algorithms for ACS include early coronary angiography, antiplatelets and antithrombotic therapy. Factors suggestive of ACS include ischemic chest pain and dynamic changes in troponin levels and/ or ECG abnormalities.

Also hemodynamic evaluation can aid one to assess severity of AHF and overall prognosis as the Killip score [12] or the Stevenson classification [13]. The latter classifies the patients into hemodynamic profiles based on the absence or presence of congestion ("wet" versus "dry") and the adequacy of peripheral perfusion ("warm" versus "cold") [13]. In addition, a focused $A B C$ assessment of the Airway/Breathing (ventilation, oxygenation) and the Circulation (stable ("warm" state) or non-stable with low blood pressure/signs of inadequate systemic perfusion ("cold" state)) might be crucial to guide the early and proper maagement (Figure 1). Following airway and oxygenation assessment, initial stabilization includes prompt correction of hemodynamic and intravascular volume abnormalities.

\section{Therapy}

\section{Treat the trigger}

Patients with AHF often suffer from significant coronary artery disease even in the absence of ACS. In patients with ST elevation myocardial infarction (STEMI) immediate revascularization is mandatory $[14,15]$. In patients with non-STEMI ACS and AHF, urgent $(<2 \mathrm{~h})$ revascularization is recommended by current guidelines $[16,17]$. Revascularization by percutaneous coronary intervention $(\mathrm{PCl})$ and coronary bypass surgery have been proven to reduce mortality in cardiogenic shock subsequent to ACS $[5,6]$, and the patient should be referred for coronary angiogram without delay.

Tachycardia as precipitant of acute decompensated heart failure should be terminated pharmacologically (hemodynamically stable patient) or by electrical cardioversion (hemodynamically compromised patient). Vice versa, bradycardia may require urgent implantation of a (temporary) pacemaker.

\section{Secure oxygenation}

Sufficient oxygenation is mandatory $\left(\mathrm{SaO}_{2}>\right.$ $90 \%$ ), may require supplementation of additional oxygen, and early implementation of non-invasive positive pressure ventilation (NIV) is encouraged. However, a previously postulated benefit of NIV regarding a reduction in mortality or reduced rate of endotracheal intubation could not be confirmed in a recent randomized controlled study [18]. Besides the general indication for endotracheal intubation (physical exhaustion, dimin-

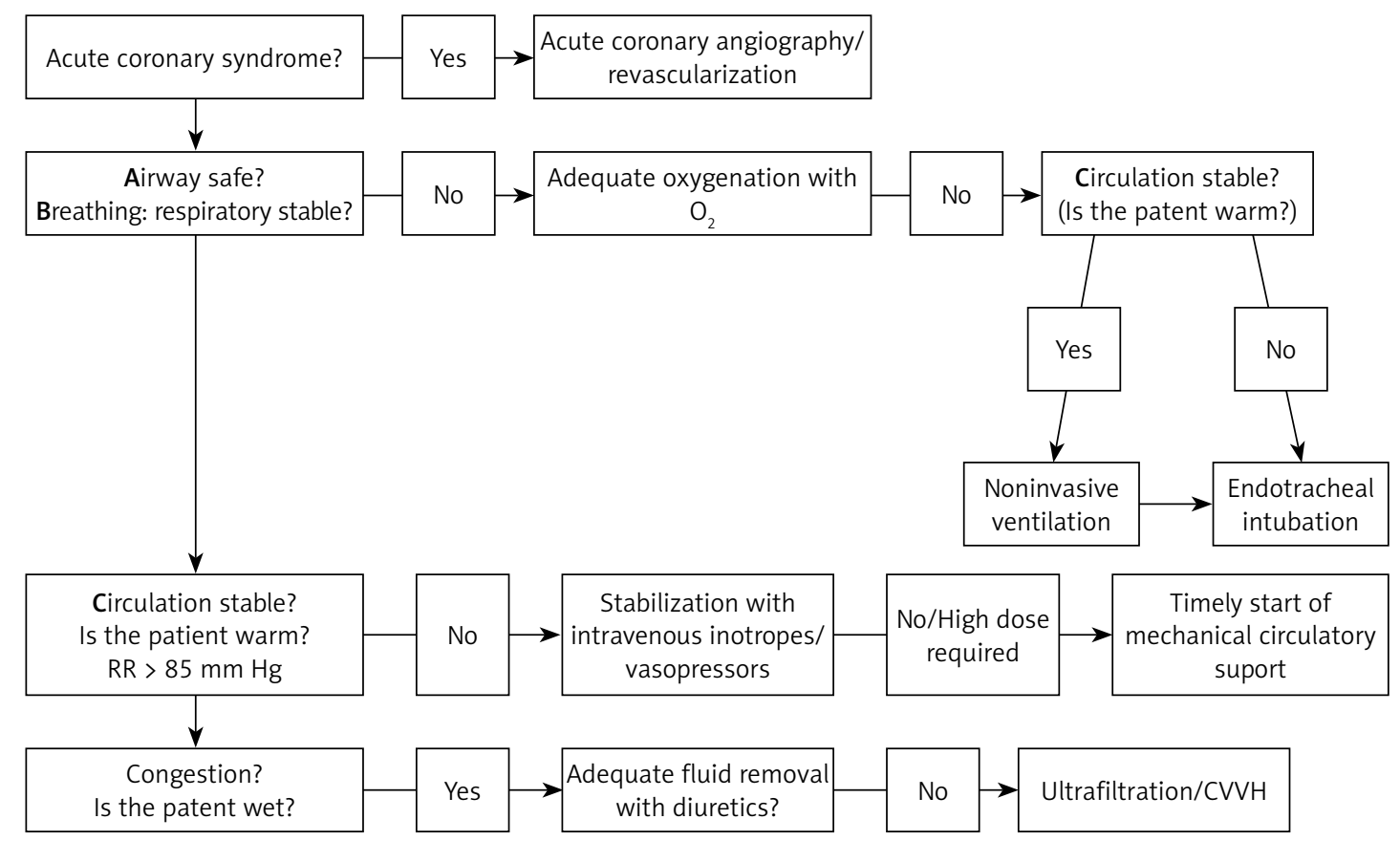

Figure 1. Algorithm for integration of medical therapy and mechanical circulatory support in management of acute heart failure. Following airway and oxygenation assessment, initial stabilization includes initiation of rapid correction of hemodynamic and intravascular volume abnormalities $\mathrm{CVVH}$ - continuous veno-venous hemofiltration. 
ished consciousness) endotracheal intubation and conventional mechanical ventilation should be considered in case of insufficient oxygenation and hemodynamic instability (e.g. a systolic blood pressure $<85 \mathrm{~mm} \mathrm{Hg}$ ).

\section{Is the patient "warm" or "cold"? - Preservation of circulation for adequate perfusion}

\section{"Warm" profile, systolic blood pressure $\geq 110 \mathrm{~mm} \mathrm{Hg}$}

In patients with a "warm" hemodynamic profile and preserved circulation, vasodilators such as nitroglycerine reduce cardiac preload and afterload, enhance stroke volume and are therefore preferred medications to offload the heart. Serelaxin as a recombinant vasoactive pregnancy hormone presents a new pharmacological option in the management of AHF with a mainly vasodilative effect. In the RELAX-AHF trial, an infusion of serelaxin over $24 \mathrm{~h}$ in patients with AHF and a systolic blood pressure $>125 \mathrm{~mm} \mathrm{Hg}$ reduced dyspnea and hospital length of stay [19]. As a secondary endpoint, a reduction in mortality over 6 months could be demonstrated, potentially related to pleiotropic effects (inhibition of apoptosis or inflammation). However, these promising observations need to be confirmed in pre-specified further trials with a reduction in mortality being a primary endpoint.

\section{"Warm" profile, systolic blood pressure 85-110 mm Hg}

In this setting, the latest heart failure guidelines from the European Society of Cardiology (ESC) and the American College of Cardiology Foundation (ACCF)/American Heart Association (AHA) Task Force recommend neither the use of vasodilators nor the use of inotropes (IIIC) [11, 20]. The latter recommendation is based on concerns about myocardial ischemia and cardiac arrhythmias being triggered by inotropic agents.

\section{"Cold" profile, systolic blood pressure $<85 \mathrm{~mm} \mathrm{Hg}$ or signs of inadequate perfusion}

In patients with AHF presenting with a "cold" hemodynamic profile, intravenous inotropes should be administered according to the ESC and ACCF/AHA guidelines to enhance cardiac contractility and to improve systemic perfusion [11, 20]. However, until now inotropes have failed to improve prognosis of AHF. Instead, there are concerns about the side effects mentioned above, which may increase mortality [21-23].

The most commonly used intravenous inotropes include the $\beta$-adrenergic agonist dobutamine, the phosphodiesterase inhibitor milrinone and (available in Europe) the calcium-sensitizing agent levosimendan as an add-on therapy for AHF refractory to standard treatment. Milrinone and levosimendan are potent vasodilators ("inodilators"), reducing mean arterial pressure to a greater extent than dobutamine and thus bearing the risk of hypotensive episodes, making continuous blood pressure monitoring necessary [24, 25]. Trials comparing inotropes (levosimendan vs. dobutamine) failed to show superiority of a single agent [25]: Whereas short-term intravenous infusions of levosimendan exerted superior hemodynamic effects compared to dobutamine, the association with lower mortality in secondary and post hoc analyses could not be confirmed in a prospective setting by the Survival of Patients with Acute Heart Failure in Need of Intravenous Inotropic Support Study (SURVIVE) evaluating long-term survival [26].

Consequently, the guidelines do not emphasize a particular inotrope, although dobutamine is recommended in the ESC guidelines receiving evidence grade Ila, while levosimendan and milrinone receive evidence grade IIb [11]. The latter may be considered in conditions where $\beta$-blockade is thought to contribute to hypoperfusion by blunted dobutamine response.

If blood pressure cannot be maintained with inotropes, a vasopressor may be considered. In the ESC guidelines norepinephrine and dopamine (at higher doses of $10-20 \mu \mathrm{g} / \mathrm{kg} \mathrm{min}$ ) are recommended at equivalent evidence grade (IIb) [11], although one study has demonstrated inferiority of dopamine over norepinephrine due to more arrhythmic events [27]. The drawbacks of the use of vasopressors are increased heart rate and myocardial oxygen demand as well as tachyarrhythmias. The intended elevation in systemic vascular resistance to maintain the blood pressure increases cardiac afterload and the risk of myocardial ischemic injury.

\section{Mechanical circulatory support (MCS) in cardiogenic shock}

In addition to medical therapy, circulatory assist systems can be used in patients with fulminant cardiogenic shock for hemodynamic stabilization and maintenance of perfusion until the underlying cause of shock has been reversed (bridge to recovery) or until the definite decision regarding a permanent supply (bridge to decision) is made.

The intra-aortic balloon pump (IABP) is implanted percutaneously with a balloon placed in the descending thoracic aorta. Inflation of the balloon in diastole and deflation in systole induce higher diastolic blood pressure, an improvement of coronary perfusion and unloading of the left ventricle by reducing cardiac afterload. Whereas the benefi- 
cial effect with regard to increased cardiac output is only marginal, reduction of cardiac afterload is effective for unloading the left ventricle especially in states of relevant mitral regurgitation.

However, various studies have challenged the benefit of IABP. A meta-analysis demonstrated divergent findings regarding a beneficial effect of IABP [28-30] on outcome, potentially flawed by the heterogeneity of the patients included and by publication bias leading to overrepresentation of studies with positive findings regarding IABP effectiveness. The ESC and ACCF/AHA guidelines of treatment of patients with STEMI (2012) recently downgraded the class of recommendation and level of evidence for IABP use in acute myocardial infarction complicated by cardiogenic shock as class Ila (ACCF/AHA) [15] or IIb (ESC) [14].

Recently, the only sufficiently powered randomized trial of IABP counterpulsation in cardiogenic SHOCK secondary to myocardial infarction (IABP-SHOCK II) did not show any beneficial effects of IABP support on 30-day or 12-month survival in patients with cardiogenic shock receiving early revascularization and optimal medical therapy [4]. The results of the IABP-SHOCK II trial endorse the further downgrading of IABP use in this setting.

The IABP support may not provide adequate active support in severe cardiogenic shock with profound low output. Today, potent circulatory assist systems are available allowing organ perfusion even at stages of minimal endogenous myocardial contractility or during prolonged functional cardiac arrest (e.g. ventricular fibrillation):

The axial flow pump (e.g. Impella) is placed percutaneously via the femoral artery crossing the aortic valve within the left ventricle. The system aspirates blood from the left ventricle and expels it into the ascending aorta. Depending on the system implanted (the larger one requires surgical cutdown) a cardiac output of 2.5 up to $5 \mathrm{l} / \mathrm{min}$ can be provided. Favorable effects of the Impella device consist of effective unloading of the left ventricle, increased coronary perfusion, augmented cardiac output and blood pressure as well as improved organ perfusion as suggested by reduced lactate levels [31, 32]. However, so far no reduction of mortality could be demonstrated in clinical trials employing the Impella device in patients with cardiogenic shock compared to IABP [37]. No randomized controlled trial (RCT) or meta-analysis is currently availably investigating the effect of Impella implantation on mortality compared to optimal medical treatment alone.

Circulatory support with the extracorporeal life support (ECLS) device is performed with a partial cardiopulmonary bypass. Blood is drained by a centrifugal pump through a percutaneously implanted venous cannula inserted into the right atrium (e.g. via femoral vein access), oxygenated and decarboxylated over a membrane and restituted (e.g. via femoral artery access) into the systemic circulation. Reports of the use of ECLS for cardiogenic shock provide first evidence of longterm survival up to $40-60 \%$ in these patients [33, 34]. Until now, there is no RCT or meta-analysis for ECLS systems with mortality as an endpoint. However, especially the miniaturization of these ECLS devices with minimally invasive percutaneously placed cannulae and the option of effective long-term (weeks) support establishes new therapy options. The ESC and ACCF/AHA heart failure guidelines recommend the use of MCS devices as a "bridge to recovery" concept for patients with refractory cardiogenic shock and a potentially reversible cause (e.g. viral myocarditis) or a potentially surgically correctable cause (IIa) [11, 20]. In addition, MCS may be considered as a "bridge to decision" concept in patients deteriorating rapidly before a full diagnostic and clinical evaluation can be made $[11,20]$. The ESC and ACCF/AHA guidelines of treatment of patients with STEMI (2012) provide a grade $\mathrm{IIb}$ recommendation for the implantation of an MCS in patients with refractory shock [14, 15].

Regarding the limited data about the effect of MCS on outcome, MCS cannot be generally recommended as first-line treatment in cardiogenic shock but may be considered on an individual basis targeting fundamental pathophysiological aspects that are largely hemodynamic and mechanical in nature and cannot be targeted with the currently available medical therapy. We recommend that MCS should not only be considered as a further step of escalation in the therapy of refractory cardiogenic shock (high concentration of vasopressors and inotropes) but should rather be implanted at earlier stages before manifestation of awkward circulatory collapse and MODS. Early identification of impending circulatory collapse coupled with rapid implementation of MCS may contribute to mortality reduction [7].

\section{Is the patient "wet"? - From diuretics to mechanical volume removal}

Relief of congestion is one primary goal of AHF management. In patients classified as "wet" according to bedside hemodynamic profile, loop diuretics are the therapy of choice, efficiently reducing volume overload and relieving dyspnea. In the case of resistance to loop diuretics, addition of a second diuretic agent (e.g. thiazide) acting at a more distant tubular site (sequential nephron blockade) often facilitates sufficient diuresis and fluid removal especially in patients with acute-on-chronic renal failure and worsening left ventricular function (cardiorenal syndrome type I and II) [35]. Continuous 
infusion of loop diuretics is equipotent to intermittent bolus administration. Administration of highdose loop diuretics has been associated with the onset of transient worsening renal function without impairing long-term prognosis but provides faster relief of congestion and dyspnea [36].

Veno-venous ultrafiltration is an alternative or additional approach to diuretics that can successfully be applied for treatment of volume overload. Ultrafiltration removes isotonic intravasal volume. Compared to diuretics, potential benefits may consist of decreased RAAS-activation and superior sodium removal in patients with acute-on-chronic heart failure.

The UNLOAD and CARRESS-HF trials compared ultrafiltration with diuretic therapy in patients with AHF and volume overload in a randomized controlled setting. The UNLOAD trial showed superiority of ultrafiltration over diuretics with regard to fluid removal, weight loss and readmission rate at 90 days, but the results were flawed by various study limitations with respect to therapy monitoring and administration of diuretic doses [37]. In the CARRESS-HF trial no difference was observed between ultrafiltration and diuretic therapy in terms of weight loss or rehospitalization [38]. Further studies are needed to determine the ultimate role of ultrafiltration in patients with AHF. Today, ultrafiltration should be reserved as an alternative therapeutic approach for selected patients with advanced AHF who do not adequately respond to sequential nephron blockade with sufficient diuretic doses.

Tolvaptan (a vasopressin V2-receptor antagonist) may be used to treat patients with resistant hyponatremia (thirst and dehydration are recognized adverse effects) but also did not improve life quality or mortality compared to placebo [39].

\section{Supportive management}

Morphine reduces anxiety and alleviates respiratory distress, both augmenting potentially awkward vasoconstriction with increased afterload. In this regard, opiates may not only reduce sympathetic drive but also directly reduce preload due to venodilation.

\section{Conclusions}

Acute heart failure manifested as cardiogenic shock is still characterized by high mortality of up to $50 \%$. Whereas recent advances in the medical and device therapy have been implemented in guidelines and clinical practice to relieve symptoms (ultrafiltration, new vasodilators and new inotropes), neither of these innovations has reduced mortality. Until now, only early coronary revascularization in cardiogenic shock complicating myocardial infarction has improved outcome.
Safety and effectiveness of MCS devices have improved in the last years to a point proving equal to heart transplantation about at least 2 years. More RCTs are warranted with clearly defined patient populations suffering from acute heart failure and early implantation time points to investigate the prognostic benefit of MCS in selected patients.

\section{Conflict of interest}

The authors declare no conflict of interest.

\section{References}

1. Adams KF Jr, Fonarow GC, Emerman CL, et al. Characteristics and outcomes of patients hospitalized for heart failure in the United States: rationale, design, and preliminary observations from the first 100,000 cases in the Acute Decompensated Heart Failure National Registry (ADHERE). Am Heart J 2005; 149: 209-16.

2. Schocken DD, Benjamin EJ, Fonarow GC, et al. Prevention of heart failure: a scientific statement from the American Heart Association Councils on Epidemiology and Prevention, Clinical Cardiology, Cardiovascular Nursing, and High Blood Pressure Research; Quality of Care and Outcomes Research Interdisciplinary Working Group; and Functional Genomics and Translational Biology Interdisciplinary Working Group. Circulation 2008; 117: 2544-65.

3. Rudiger A, Harjola VP, Muller A, et al. Acute heart failure: clinical presentation, one-year mortality and prognostic factors. Eur J Heart Fail 2005; 7: 662-70.

4. Thiele H, Zeymer U, Neumann FJ, et al. Intra-aortic balloon counterpulsation in acute myocardial infarction complicated by cardiogenic shock (IABP-SHOCK II): final 12 month results of a randomised, open-label trial. Lancet 2013; 382: 1638-45.

5. Hochman JS, Sleeper LA, Webb JG, et al. Early revascularization in acute myocardial infarction complicated by cardiogenic shock. SHOCK Investigators. Should We Emergently Revascularize Occluded Coronaries for Cardiogenic Shock. N Engl J Med 1999; 341: 625-34.

6. White HD, Assmann SF, Sanborn TA, et al. Comparison of percutaneous coronary intervention and coronary artery bypass grafting after acute myocardial infarction complicated by cardiogenic shock: results from the Should We Emergently Revascularize Occluded Coronaries for Cardiogenic Shock (SHOCK) trial. Circulation 2005; 112: 1992-2001.

7. Takayama H, Truby L, Koekort M, et al. Clinical outcome of mechanical circulatory support for refractory cardiogenic shock in the current era. J Heart Lung Transplant 2013; 32: 106-11.

8. Rogers JG, O'Connor CM. The changing landscape of advanced heart failure therapeutics. J Am Coll Cardiol 2014; 64: 1416-7.

9. Drazner MH, Hellkamp AS, Leier CV, et al. Value of clinician assessment of hemodynamics in advanced heart failure: the ESCAPE trial. Circulation Heart Failure 2008; 1: $170-7$.

10. Dao Q, Krishnaswamy P, Kazanegra R, et al. Utility of B-type natriuretic peptide in the diagnosis of congestive heart failure in an urgent-care setting. J Am Coll Cardiol 2001; 37: 379-85.

11. McMurray J, Adamopoulos S, Anker SD, et al. ESC Guidelines for the diagnosis and treatment of acute and 
chronic heart failure 2012: The Task Force for the Diagnosis and Treatment of Acute and Chronic Heart Failure 2012 of the European Society of Cardiology. Developed in collaboration with the Heart Failure Association (HFA) of the ESC. Eur Heart J 2012; 33: 1787-847.

12. Killip T 3rd, Kimball JT. Treatment of myocardial infarction in a coronary care unit. A two year experience with 250 patients. Am J Cardiol 1967; 20: 457-64.

13. Nohria A, Tsang SW, Fang JC, et al. Clinical assessment identifies hemodynamic profiles that predict outcomes in patients admitted with heart failure. J Am Coll Cardiol 2003; 41: 1797-804.

14. Task Force on the management of STseamiotESoC, Steg PG, James SK, et al. ESC Guidelines for the management of acute myocardial infarction in patients presenting with ST-segment elevation. Eur Heart J 2012; 33: 2569-619.

15. O'Gara PT, Kushner FG, Ascheim DD, et al. 2013 ACCF/ AHA guideline for the management of ST-elevation myocardial infarction: a report of the American College of Cardiology Foundation/American Heart Association Task Force on Practice Guidelines. Circulation 2013; 127: e362-425.

16. Hamm CW, Bassand JP, Agewall S, et al. ESC Guidelines for the management of acute coronary syndromes in patients presenting without persistent ST-segment elevation: The Task Force for the management of acute coronary syndromes (ACS) in patients presenting without persistent ST-segment elevation of the European Society of Cardiology (ESC). Eur Heart J 2011; 32: 2999-3054.

17. Anderson JL, Adams CD, Antman EM, et al. 2012 ACCF/ AHA focused update incorporated into the ACCF/AHA 2007 guidelines for the management of patients with unstable angina/non-ST-elevation myocardial infarction: a report of the American College of Cardiology Foundation/American Heart Association Task Force on Practice Guidelines. Circulation 2013; 127: e663-828.

18. Gray A, Goodacre S, Newby DE, et al. Noninvasive ventilation in acute cardiogenic pulmonary edema. N Engl J Med 2008; 359: 142-51.

19. Teerlink JR, Metra M, Felker GM, et al. Relaxin for the treatment of patients with acute heart failure (PreRELAX-AHF): a multicentre, randomised, placebo-controlled, parallel-group, dose-finding phase $\mathrm{Ilb}$ study. Lancet 2009; 373: 1429-39.

20. Writing Committee M, Yancy CW, Jessup M, et al. 2013 ACCF/AHA guideline for the management of heart failure: a report of the American College of Cardiology Foundation/American Heart Association Task Force on practice guidelines. Circulation 2013; 128: e240-327.

21. Cuffe MS, Califf RM, Adams KF Jr, et al. Short-term intravenous milrinone for acute exacerbation of chronic heart failure: a randomized controlled trial. JAMA 2002; 287: 1541-7.

22. Elkayam U, Tasissa G, Binanay C, et al. Use and impact of inotropes and vasodilator therapy in hospitalized patients with severe heart failure. Am Heart J 2007; 153: 98-104.

23. Bayram M, De Luca L, Massie MB, Gheorghiade M. Reassessment of dobutamine, dopamine, and milrinone in the management of acute heart failure syndromes. Am J Cardiol 2005; 96: 47G-58G.

24. Colucci WS, Wright RF, Jaski BE, Fifer MA, Braunwald E. Milrinone and dobutamine in severe heart failure: differing hemodynamic effects and individual patient responsiveness. Circulation 1986; 73: III175-83.
25. Mebazaa A, Nieminen MS, Packer M, et al. Levosimendan vs dobutamine for patients with acute decompensated heart failure: the SURVIVE Randomized Trial. JAMA 2007; 297: 1883-91.

26. Follath F, Cleland JG, Just H, et al. Efficacy and safety of intravenous levosimendan compared with dobutamine in severe low-output heart failure (the LIDO study): a randomised double-blind trial. Lancet 2002; 360: 196-202.

27. De Backer D, Biston P, Devriendt J, et al. Comparison of dopamine and norepinephrine in the treatment of shock. N Engl J Med 2010; 362: 779-89.

28. Sjauw KD, Engstrom AE, Vis MM, et al. A systematic review and meta-analysis of intra-aortic balloon pump therapy in ST-elevation myocardial infarction: should we change the guidelines? Eur Heart J 2009; 30: 459-68.

29. Unverzagt S, Machemer MT, Solms A, et al. Intra-aortic balloon pump counterpulsation (IABP) for myocardial infarction complicated by cardiogenic shock. Cochrane Database Syst Rev 2011; 7: CD007398.

30. Bahekar A, Singh M, Singh S, et al. Cardiovascular outcomes using intra-aortic balloon pump in high-risk acute myocardial infarction with or without cardiogenic shock: a meta-analysis. J Cardiovasc Pharmacol Ther 2012; 17: 44-56.

31. Ouweneel DM, Henriques JP. Percutaneous cardiac support devices for cardiogenic shock: current indications and recommendations. Heart 2012; 98: 1246-54.

32. Seyfarth M, Sibbing D, Bauer I, et al. A randomized clinical trial to evaluate the safety and efficacy of a percutaneous left ventricular assist device versus intra-aortic balloon pumping for treatment of cardiogenic shock caused by myocardial infarction. J Am Coll Cardiol 2008; 52: 1584-8.

33. Jaski BE, Lingle RJ, Overlie $P$, et al. Long-term survival with use of percutaneous extracorporeal life support in patients presenting with acute myocardial infarction and cardiovascular collapse. ASAIO J 1999; 45: 615-8.

34. Kim H, Lim SH, Hong J, et al. Efficacy of veno-arterial extracorporeal membrane oxygenation in acute myocardial infarction with cardiogenic shock. Resuscitation 2012; 83: 971-5.

35. Jentzer JC, DeWald TA, Hernandez AF. Combination of loop diuretics with thiazide-type diuretics in heart failure. J Am Coll Cardiol 2010; 56: 1527-34.

36. Felker GM, Lee KL, Bull DA, et al. Diuretic strategies in patients with acute decompensated heart failure. N Engl J Med 2011; 364: 797-805.

37. Costanzo MR, Saltzberg MT, Jessup M, Teerlink JR, Sobotka PA. Ultrafiltration versus intravenous diuretics for patients hospitalized for acute decompensated heart failure I. Ultrafiltration is associated with fewer rehospitalizations than continuous diuretic infusion in patients with decompensated heart failure: results from UNLOAD. J Card Fail 2010; 16: 277-84.

38. Bart BA, Goldsmith SR, Lee KL, et al. Ultrafiltration in decompensated heart failure with cardiorenal syndrome. N Engl J Med 2012; 367: 2296-304.

39. Gheorghiade M, Konstam MA, Burnett JC Jr, et al. Shortterm clinical effects of tolvaptan, an oral vasopressin antagonist, in patients hospitalized for heart failure: the EVEREST Clinical Status Trials. JAMA 2007; 297: 1332-43. 